\title{
Nonequilibrium localization in quantum Hall systems at very low frequencies
}

\author{
A. Buss, ${ }^{1}$ F. Hohls,${ }^{2}$ F. Schulze-Wischeler, ${ }^{2}$ C. Stellmach, ${ }^{1}$ G. Hein, ${ }^{3}$ R. J. Haug, ${ }^{2}$ and G. Nachtwei ${ }^{1, *}$ \\ ${ }^{1}$ Institut für Angewandte Physik, Technische Universität Braunschweig, Mendelssohnstrasse 2, D-38106 Braunschweig, Germany \\ ${ }^{2}$ Institut für Festkörperphysik, Universität Hannover, Appelstrasse 2, D-30167 Hannover, Germany, \\ ${ }^{3}$ Physikalisch-Technische Bundesanstalt, Bundesallee 100, D-38116 Braunschweig, Germany
}

(Received 1 September 2004; revised manuscript received 12 November 2004; published 20 May 2005)

\begin{abstract}
We have measured the conductivity $\sigma_{x x}$ of GaAs/GaAlAs Corbino devices under nonequilibrium conditions in the quantum Hall regime at low frequencies from dc up to $10 \mathrm{kHz}$. At very low frequencies up to $10-20 \mathrm{~Hz}$, $\sigma_{x x}$ shows a steep decrease from its dc value towards a reduced saturation value. Measurements of the temperature dependence of $\sigma_{x x}$ from $70 \mathrm{mK}$ to $4.2 \mathrm{~K}$ revealed a dominance of a conductivity with a temperature dependence $\sigma_{x x} \sim \exp \left\{-\left(T_{0} / T\right)^{1 / 2}\right\}$ typical for variable-range hopping (VRH) at temperatures below $1 \mathrm{~K}$ and an additional contribution of thermal activation above $2 \mathrm{~K}$. The characteristic temperature $T_{0}$ as determined from the VRH-like temperature plot of the conductivity showed also an increase with the frequency and a pronounced decrease with increasing voltage below the breakdown of the quantum Hall effect (QHE). We attribute these results to an effective suppression of the delocalization of electrons in alternating external fields due to a reduced heating and scattering between localization centers. The time scale for this process can be as long as $100 \mathrm{~ms}$. Our data show that the background conductivity $\sigma_{x x}$ at subcritical voltages affects the critical voltage for the breakdown of the QHE.
\end{abstract}

DOI: $10.1103 /$ PhysRevB.71.195319

PACS number(s): 73.43.Fj, 73.43.Cd, 73.50.Gr

\section{INTRODUCTION}

The conductivity mechanisms in Landau-quantized systems were widely investigated both experimentally and theoretically for the last three decades. In particular, the relation between the localization length $\xi$ and the effective scattering length $L_{\Phi}$ near the mobility edges of the Landau level (i.e., in the plateau-transition region) was addressed in the scaling theory. ${ }^{1-5}$ Various experiments were dedicated to the universality of the scaling exponent (see, e.g., Refs. 6-8). Measurements of the transition region between quantum Hall $(\mathrm{QH})$ plateaus up to frequencies in the $\mathrm{GHz}$ range revealed the universality of scaling exponents up to these frequencies, concomitant with a reduction of the plateau width at higher frequencies. ${ }^{9,10}$ In the plateau centers, measurements of $\xi$ are generally difficult, due to the low values of $\sigma_{x x}$ in the $\mathrm{QH}$ regime. However, at higher frequencies $\xi$ has been measured recently, showing values of the localization length in the plateau centers approaching the magnetic length. ${ }^{11}$ At higher temperatures-or driving voltages-respectively, it is possible to obtain measurable values of $\sigma_{x x}$ at integer-filling factors (e.g., Ref. 12). It was shown that a monotonous increase of $\sigma_{x x}$ with the voltage (of $\rho_{x x}$ with the sample current) occurs before the quantum Hall effect (QHE) finally breaks down. It is a long-standing question how far the breakdown of the QHE itself is related to this increase of $\sigma_{x x}$ in the subcritical regime. In earlier papers, a direct relation between the critical value of the voltage or current and the subcritical values of $\sigma_{x x}$ or $\rho_{x x}$ was postulated ${ }^{12-14}$ in the framework of a phenomenological hot-electron model, whereas this relation was excluded in a model presented in a more recent paper. ${ }^{15}$

Usually, the conductivity in subcritical QH systems is attributed to variable-range hopping (VRH) for low temperatures below $1 \mathrm{~K}$ (see, e.g., Refs. 16-18). Increasing the temperature, thermal activation (TA) over the Landau gap begins to contribute and finally dominates the temperature dependence of $\sigma_{x x}{ }^{19-21}$ At intermediate temperatures $1 \mathrm{~K}<T$ $<4.2 \mathrm{~K}$, it was shown that both TA and VRH can coexist. ${ }^{22}$

In this study, we have investigated the conductivity $\sigma_{x x}$ of $\mathrm{QH}$ devices in Corbino geometry as a function of the temperature between $70 \mathrm{mK}$ and $4.2 \mathrm{~K}$, of the source-drain voltage below the critical value, and of the frequency from dc up to $10 \mathrm{kHz}$. At very low frequencies below $20 \mathrm{~Hz}$, the measured values of the nonequilibrium $\sigma_{x x}$ drop steeply from their dc value to a saturation value, which is reached between 10 and $20 \mathrm{~Hz}$ and persists until some kHz. Thus, the localization under nonequilibrium conditions is stronger, and hence the stability of the QHE is higher compared to the dc case. This is a rather unexpected result in view of the recent findings concerning the ac $\mathrm{QHE}$ at $\mathrm{kHz}$ frequencies: precision measurement revealed an increasing loss of precision of the $\mathrm{QH}$ quantization, in particular at the plateau flanks. ${ }^{23}$

A low-frequency decay of the conductivity $\sigma_{x x}$ under nonequilibrium conditions (further called nonequilibrium conductivity) was predicted in earlier papers to explain the steep increase of the maximum breakdown voltage and of the dynamical breakdown hysteresis, as observed in oscillation generators based on Corbino QH devices..$^{24,25}$ In our previous paper, ac measurements of the I-V characteristics of the $\mathrm{QH}$ breakdown in Corbino devices directly revealed higher upper limits of the breakdown hysteresis at frequencies of some $\mathrm{Hz}$ as compared to the dc curves. ${ }^{25}$ Thus, the measurements in this study of the frequency dependence of the nonequilibrium conductivity $\sigma_{x x}$ give direct experimental evidence for the predicted decrease of $\sigma_{x x}$ with increasing frequency, and that $\sigma_{x x}$ at subcritical voltages dominantly determines the critical voltage for the breakdown of the QHE. With this, our measurements cannot be explained by a model, postulating a breakdown of the QHE independent of the prebreakdown evolution of the conductivity. ${ }^{15}$ Instead, our measurements confirm the prediction of the hot-electron model (as devel- 
oped in Ref. 25) for the relation between the breakdown of the QHE and the nonequilibrium conductivity $\sigma_{x x}$ in the prebreakdown (subcritical) regime. Further, we found a dominance of conductivity with a temperature dependence $\sigma_{x x}$ $\sim \exp \left\{-\left(T_{0} / T\right)^{1 / 2}\right\}$ that is typical for variable-range hopping (VRH) at temperatures below $1 \mathrm{~K}$ and an additional contribution of thermal activation above $2 \mathrm{~K}$. With this, our results show that also very low energy excitations (of the order of $k_{\mathrm{B}} T$ ) can determine the breakdown, not only the interband excitations (of the order of $\hbar \omega_{\mathrm{c}}$ ).

\section{EXPERIMENTAL DETAILS}

On two wafers of GaAs/GaAlAs heterojunctions (wafer $A$ : electron density $n_{\mathrm{s}}=2.7 \times 10^{11} \mathrm{~cm}^{-2}$, Hall mobility $\mu_{\mathrm{H}}$ $=1.0 \times 10^{5} \mathrm{~cm}^{2} / \mathrm{V} \mathrm{s}$; wafer $B: n_{\mathrm{s}}=4.8 \times 10^{11} \mathrm{~cm}^{-2}, \mu_{\mathrm{H}}=1.8$ $\times 10^{5} \mathrm{~cm}^{2} / \mathrm{V} \mathrm{s}$ ), Corbino samples with an inner contact radius $r_{\mathrm{i}}$ of $100 \mu \mathrm{m}$ and three different radii $r_{\mathrm{a}}$ of the outer contact of 150,200 , and $300 \mu \mathrm{m}$ (i.e., three widths of the two-dimensional (2D) channel of 50,100, and $200 \mu \mathrm{m}$ ) were patterned (accordingly we refer to the samples as $A 100, B 50$, etc.). All samples were characterized by dc Shubnikov-de Haas measurements and by I-V characteristics. [For the values $V_{\min }$ and $V_{\max }$, limiting the hysteresis of the QH breakdown in the dc regime, see, e.g., the inset in Fig. 1(a).]

From the enhanced amplitude of the relaxation oscillations previously observed in the bistable regime of the $\mathrm{QH}$ breakdown in Corbino devices, ${ }^{24}$ we deduced an enhanced dynamical hysteresis of the breakdown. Therefore, we confirmed this by taking the frequency dependence of the I-V curves at low ac frequencies and found a steep increase of the hysteresis in the frequency range from dc up to about $20 \mathrm{~Hz}$ (for details, see Ref. 25). This behavior was consistent for all samples used in this study (see a typical example in Fig. 1).

\section{RELATION BETWEEN THE NONEQUILIBRIUM CONDUCTIVITY $\sigma_{x x}$ AND THE BREAKDOWN OF THE QHE: THE HOT-ELECTRON MODEL WITH BACKGROUND CONDUCTIVITY}

An explanation of the existence of the hysteresis of the current-voltage characteristics of $\mathrm{QH}$ devices at the breakdown of the quantum Hall effect can be derived from the hot-electron model (HEM). ${ }^{12,13}$ In this model, the hysteresis arises from the metastability of the power balance between gain (electrical energy fed to the system per time and area), $p_{\text {gain }}=\sigma_{x x}\left(T_{\mathrm{el}}\right) E_{\mathrm{r}}^{2}\left(\sigma_{x x}\right.$ is the longitudinal conductivity of the $\mathrm{QH}$ system, $E_{\mathrm{r}}$ is the radial electric field in the Corbino device), and the energy-loss rate $p_{\text {loss }}=\left[\varepsilon\left(T_{\mathrm{el}}\right)-\varepsilon\left(T_{\mathrm{L}}\right)\right] / \tau_{\text {rel }}$, which describes the relaxation of the energy per area $\varepsilon\left(T_{\mathrm{el}}\right)$ at the elevated electron temperature $T_{\text {el }}$ back to the energy per area $\varepsilon\left(T_{\mathrm{L}}\right)$ at the lattice temperature $T_{\mathrm{L}}$. The relaxation time $\tau_{\text {rel }}$ depends on the electron temperature for scattering at phonons ${ }^{13}$ and, as shown later, by scattering at impurities. ${ }^{14,26}$

The energy per area $\varepsilon(T)$ of the electron system as a function of temperature can be analytically calculated for the Fermi energy $E_{\mathrm{F}}$ situated in the middle of the Landau gap, if
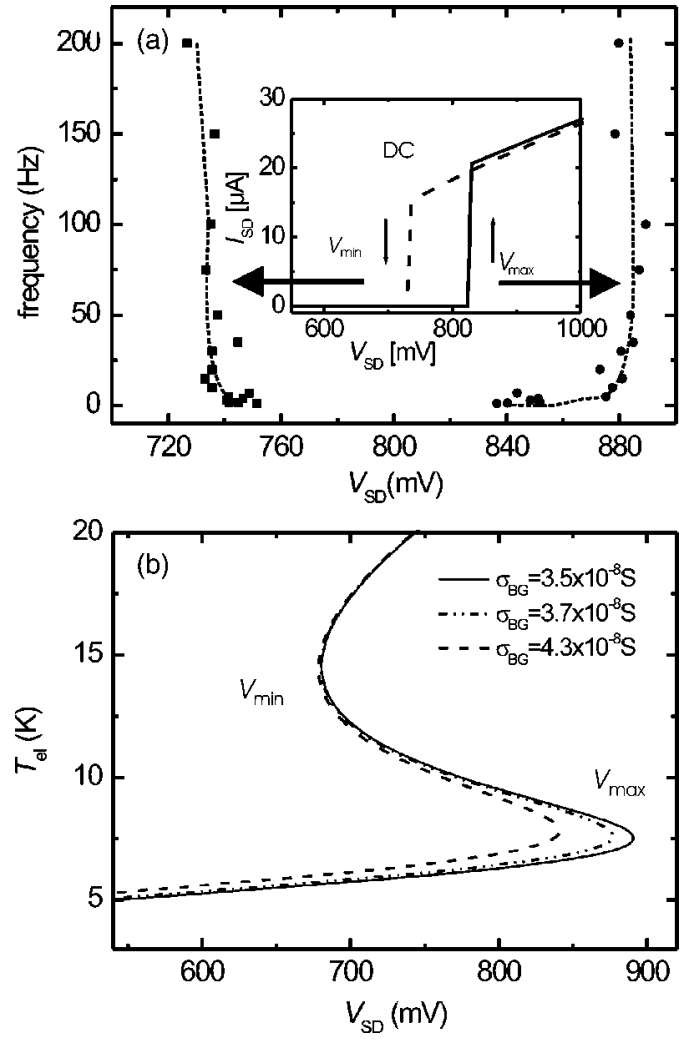

FIG. 1. Dynamic hysteresis of the QH breakdown as a function of the frequency (sample $A 100, \nu=2$ ). (a) Separate plot of the hysteresis limits $V_{\max }$ and $V_{\min }$ as a function of the frequency. Inset: corresponding I-V characteristics (dc), showing the hysteresis in the zero-frequency limit. (b) Modeling of the change of the hysteresis, as observed for sample $A 100$, by a variation of the background conductivity $\sigma_{\mathrm{BG}}$ (hot-electron model, see text).

there is a constant background density of (localized) states $D_{\mathrm{BG}}$, and if the relation $k_{\mathrm{B}} T \ll \hbar \omega_{\mathrm{c}}$ holds $\left(k_{B} T\right.$ : thermal energy, $\hbar \omega_{\mathrm{c}}$ : cyclotron energy). This is valid up to temperatures of $12-15 \mathrm{~K}$ for our samples, whereas the breakdown value $V_{\max }$ is typically determined by electron temperatures of about 5-7 K. Thus, low-energy excitations of the order of $k_{\mathrm{B}} T$ within the localized states of the density $D_{\mathrm{BG}}$ determine the value of $V_{\max }$ within this approach. In contrast, a recent model attributes the breakdown to excitations at the higher energy of the order of $\hbar \omega_{\mathrm{c}}$ only, regarding $D_{\mathrm{BG}}$ as unimportant in this respect. ${ }^{15}$ An essential aim of this study is to confirm the validity of the approach considering low-energy excitations within localized states of the density $D_{\mathrm{BG}}$ as given above for the samples investigated in our experiments.

From the power-balance equation, the relation between the radial electric field and the electron temperature can be evaluated, if the temperature dependence of the conductivity $\sigma_{x x}$ is known,

$$
\sigma_{x x}\left(T_{\mathrm{el}}\right)=\sigma_{0} \exp \left\{-\Delta / k_{\mathrm{B}} T_{\mathrm{el}}\right\}+\sigma_{\mathrm{BG}}
$$

The first term in the sum of Eq. (1) describes the thermal activation (the activation gap is $\Delta=\hbar \omega_{\mathrm{c}} / 2$ ), and the second term $\sigma_{\mathrm{BG}}$ is a background conductivity, which can be attrib- 


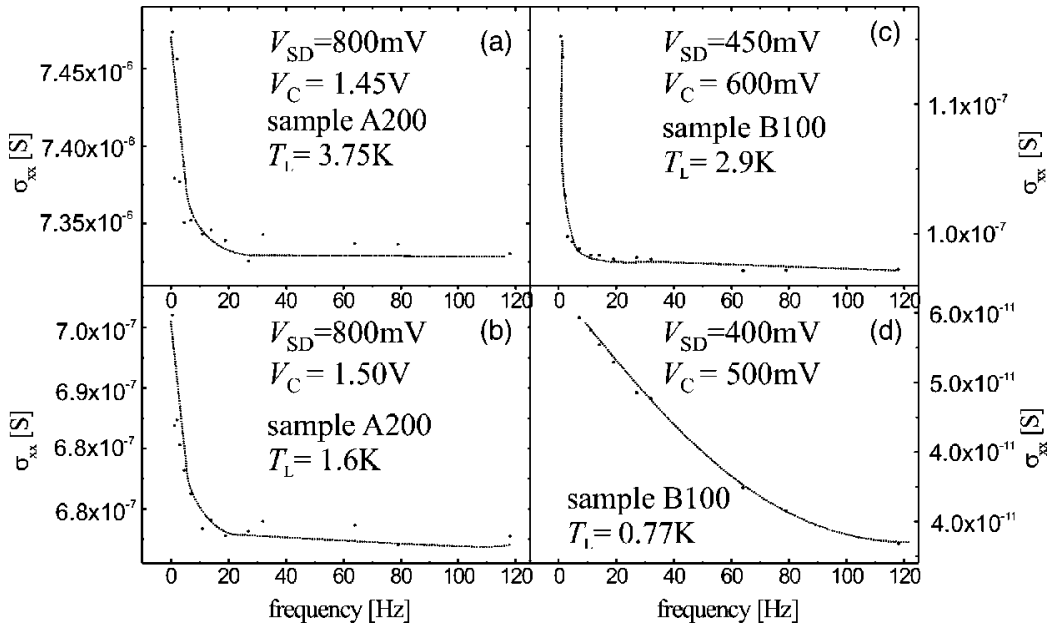

FIG. 2. Longitudinal conductivity $\sigma_{x x}$ as a function of the frequency for the samples $A 200$ (a) and (b) and $B 100$ (c) and (d), measured at different temperatures (see legend) at filling factor $\nu=2$. The values of $V_{\mathrm{SD}}$ (amplitude of the ac voltage) are set well below the critical value $V_{\mathrm{C}}$ of the dc-QH breakdown (see legend). uted, for example, to hopping (see also Ref. 14). Whereas the first term dominates at higher temperatures above about $12 \mathrm{~K}$ and determines $V_{\min }$, the background conductivity $\sigma_{\mathrm{BG}}$ dominates $V_{\max }$ at electron temperatures of about 5-7 K [see Fig. 1(b)]. Thus, a neglecting of $\sigma_{\mathrm{BG}}\left(\text { or } D_{\mathrm{BG}}\right)^{15}$ has no essential influence on the determination of $V_{\min }$, but leads to values for $V_{\max }$ being too small for our samples (see below).

With an increase in $\sigma_{\mathrm{BG}}, V_{\max }$ exhibits a pronounced decrease [see Fig. 1(b)] up to the complete disappearance of the hysteresis $\left(V_{\max } \approx V_{\min }\right)$ at $\sigma_{\mathrm{BG}}=1.3 \times 10^{-7} \mathrm{~S}=1.7 \times 10^{-3} \sigma_{x y}$. If we attribute $\sigma_{\mathrm{BG}}$ to a hopping conductivity, we can formulate $\sigma_{\mathrm{BG}}$ as follows for variable-range hopping (VRH): ${ }^{16-18,27}$

$$
\begin{gathered}
\sigma_{\mathrm{BG}}=\sigma_{0}(T) \exp \left\{-\left(\frac{T_{0}}{T}\right)^{1 / 2}\right\} \text { with } \\
\sigma_{0}(T)=S_{0} / T \text { and } \\
T_{0}=\frac{C_{\mathrm{hop}} e^{2}}{k_{\mathrm{B}} 4 \pi \varepsilon \varepsilon_{0} \xi}
\end{gathered}
$$

$\left(S_{0}\right.$ : constant conductivity prefactor; $T_{0}$ : characteristic temperature; $C_{\text {hop }}$ : hopping constant). The temperature exponent $\frac{1}{2}$ for variable-range hopping is, in contrast to the exponent $[1 /(1+d)] d$ dimensionality of the system) of Mott hopping, ${ }^{28}$ independent of the dimensionality and related to a soft Coulomb gap near the Fermi energy. ${ }^{29}$

The essential parameter for $\sigma_{\mathrm{BG}}$ is the localization length $\xi$, if hopping conductivity is assumed to be the essential mechanism. An increase in the localization length (decrease of $T_{0}$ ) yields, in accordance with Eq. (2), an increase in the hopping conductivity. Consequently, from the observed increase of the hysteresis with the frequency as reported in Ref. 25 a reduction of $\sigma_{\mathrm{BG}}$ was predicted, accompanied by an increase in $T_{0}$. In general, an increase in $T_{0}$ can be related to a decrease in $\xi$, meaning a more effective localization. However, it is questionable how far Eq. (2b), which was deduced in the linear-response limit, can be applied quantitatively at rather high voltages near the breakdown of the QHE. Therefore, in this study we have measured the conductivity $\sigma_{x x}$ as a function of the bias voltage, frequency, and temperature. Our data show clearly a trend of a suppressed delocalization of electrons already at low frequencies.

\section{EXPERIMENTAL RESULTS AND DISCUSSION}

As discussed previously, ${ }^{25}$ the HEM with background conductivity predicts that the steep increase in the critical voltage $V_{\max }$ of the $\mathrm{QH}$ breakdown observed at low frequencies is due to a reduction of the longitudinal conductivity $\sigma_{x x}$ by about $1-10 \times 10^{-8} \mathrm{~S}$ (depending on the sample properties).

Fig. 2 shows the dependence of $\sigma_{x x}$ on the frequency up to $120 \mathrm{~Hz}$, as measured on the samples $A 200$ [Figs. 2(a) and 2(b)] and $B 100$ [Figs. 2(c) and 2(d)] at different temperatures (3.75 K and $1.6 \mathrm{~K}$ for $A 200 ; 2.9 \mathrm{~K}$ and $0.77 \mathrm{~K}$ for $B 100$ ). The low-frequency drop of $\sigma_{x x}$ (from dc to $10-20 \mathrm{~Hz}$ ) was found between $2 \times 10^{-8} \mathrm{~S}$ [see Fig. 2(c)] and $1.4 \times 10^{-7} \mathrm{~S}$ [Fig. 2(a)] at temperatures above $1.5 \mathrm{~K}$. (Please note that $\sigma_{x x}$ is $4-5$ orders smaller at $0.77 \mathrm{~K}$ [Fig. 2(d)], measured above $f \geqslant 7 \mathrm{~Hz}$ with a highly sensitive amplifier, which made it, however, impossible to resolve the far steeper change of $\sigma_{x x}$ at frequencies $f<7 \mathrm{~Hz}$ in this case).

Consequently, the measured decrease in the nonequilibrium values of $\sigma_{x x}$, which occurs in the same frequency range as the previously measured increase in the dynamical hysteresis of the QHE breakdown, directly confirms the prediction of Ref. 25. This is straight experimental evidence that $\sigma_{x x}$ at subcritical voltages $\left(V_{\mathrm{SD}}<V_{\text {min }}\right)$ directly determines the breakdown of the QHE.

Further, the fact that the HEM provides a decrease of $\sigma_{x x}$ which is required to explain the increase of the critical voltage $V_{\max }$ in agreement with our measurements, proves that $V_{\max }$ is determined by the contribution $\sigma_{\mathrm{BG}}$ [Eq. (1)], which is dominant at lower temperatures $\left(T_{\mathrm{el}} \approx 6 \mathrm{~K}\right)$. For these temperatures, the thermal energy of the electrons $k_{\mathrm{B}} T$ $\approx 0.5 \mathrm{meV}$ is about a factor of 10 smaller than $\hbar \omega_{\mathrm{c}} / 2$. Therefore, for typical sample parameters $\left(\hbar \omega_{\mathrm{c}} \approx 10 \mathrm{meV}\right.$, Landaulevel broadening $\Gamma \approx 0.035 \hbar \omega_{\mathrm{c}}$ and density of localized states $D_{\mathrm{BG}} \approx 0.2 \mathrm{~m}^{*} / \pi \hbar^{2}$ ) the contribution of the thermal activation to the enhanced energy per area $\varepsilon\left(T_{\mathrm{el}}\right)$ is approximately by a factor of $10^{2}$ smaller than the contribution of the 

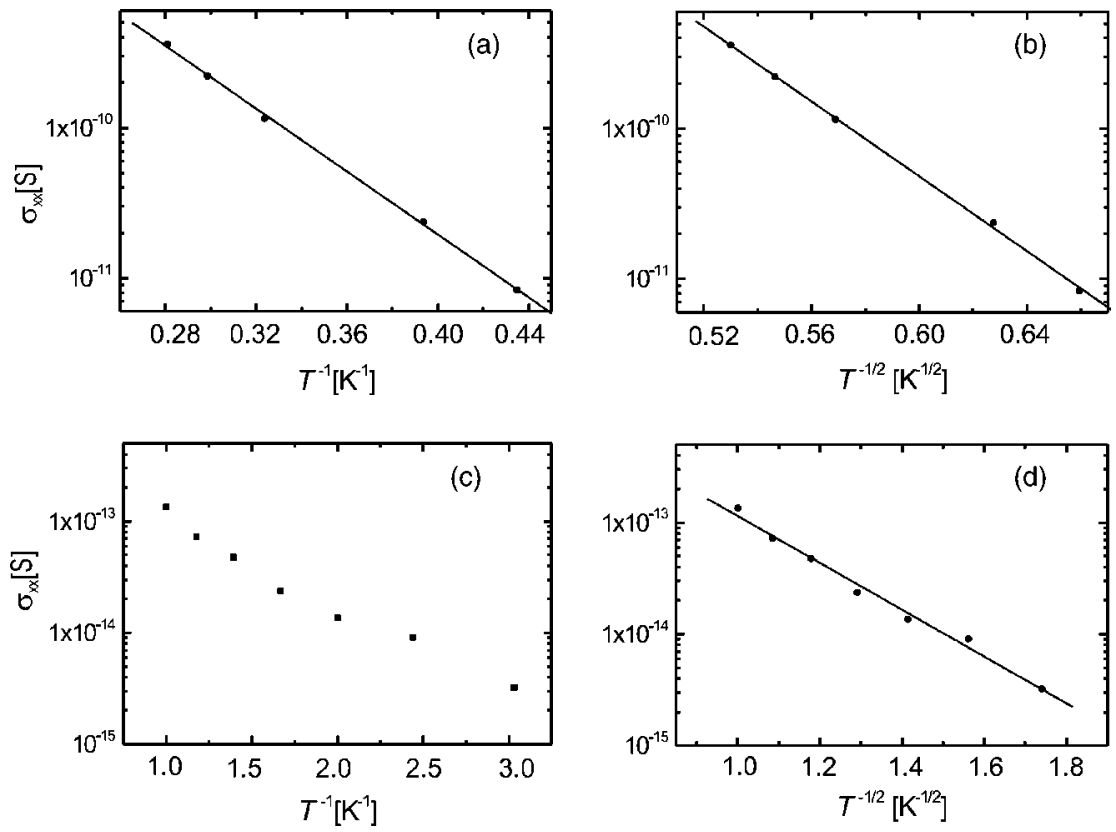

FIG. 3. Longitudinal conductivity $\sigma_{x x}(\mathrm{dc})$ at filling factor $\nu$ $=2$ as a function of the temperature. (a) Arrhenius plot $\sigma_{x x}$ $=f(1 / T), \quad$ sample A200, $\quad V_{\mathrm{SD}}$ $=360 \mathrm{mV}, T>2 \mathrm{~K}$. (b) VRH plot $\sigma_{x x}=f\left(1 / T^{1 / 2}\right)$ of the same data as in (a). (c) Arrhenius plot $\sigma_{x x}$ $=f(1 / T), \quad$ sample $A 100, \quad V_{\mathrm{SD}}$ $=804 \mathrm{mV}, T<1 \mathrm{~K}$. (d) VRH plot $\sigma_{x x}=f\left(1 / T^{1 / 2}\right)$ of the same data as in (c). low-energy excitations ( $2 k_{\mathrm{B}} T$ around the Fermi energy) within the localized states, characterized by $D_{\mathrm{BG}}$. This means, that the value $V_{\max }$ is determined by low-energy transitions (between localized states in the vicinity of the Fermi energy). This is a rather unexpected result, as the breakdown of the QHE is usually attributed to higher energy differences (from the localized states near the Fermi energy to the next higher extended state-for an overview, see Ref. 30). In particular, in a recent paper $^{15}$ the authors concluded that the breakdown does not essentially depend on the density of localized states $D_{\mathrm{BG}}$ or on hopping contributions $\sigma_{\mathrm{BG}}$ to $\sigma_{x x}$. The measured change of $\sigma_{x x}$ and $V_{\max }$ with the frequency confirms that this conclusion is not applicable for our sample parameters at electron temperatures below $10 \mathrm{~K}$. Our previous calculations ${ }^{25}$ yielded a different behavior of the lower limit of the breakdown hysteresis, $V_{\text {min }}$. As is visible in Fig. $1, V_{\min }$ was found experimentally to be only weakly dependent on the frequency, in accordance with our calculations. As $V_{\text {min }}$ is determined by electron temperatures of $T_{\mathrm{el}}$ $\approx 15 \mathrm{~K}, \sigma_{\mathrm{BG}}$ is not as important in comparison to the contribution of thermal activation over the Landau gap. Therefore, the conclusion in Ref. 15, that the $\mathrm{QH}$ breakdown is dominated by transitions to the extended states (and not by $\sigma_{\mathrm{BG}}$ ), is only applicable to our samples with respect to the lower limit of the breakdown hysteresis $V_{\text {min }}$.

Although the order of magnitude of the measured change of $\sigma_{x x}$ agrees quite well with the prediction of the HEM, the measured saturation values of $\sigma_{x x}$ at $T>1.6 \mathrm{~K}$ are 1 or 2 orders higher than calculated [larger deviations at higher temperatures, see Figs. 2(a)-2(c)].

In order to explain these larger saturation values of $\sigma_{x x}$, one could in principle invoke the idea of an inhomogeneous carrier distribution and the resulting evolution of currentcarrying filaments $^{31,32}$ at the breakdown of the QHE. ${ }^{33}$ These filaments develop preferentially in sample zones with a locally enhanced $\sigma_{x x}$ (filament) $=\sigma_{h}$, whereas other parts of the sample can remain in the $\mathrm{QH}$ regime at lower values of $\sigma_{x x}$ $(\mathrm{QHE})=\sigma_{\ell}$. Thus, the breakdown, also as modeled within the
HEM, is dominated by the local properties of the filaments (i.e., by $\sigma_{h}$ ). If the filaments occupy an angular sector $\alpha$ of the Corbino device [the remaining sector $(2 \pi-\alpha)$ is at $\sigma_{\ell}$ ], a global measurement of $\sigma_{x x}=\langle\sigma\rangle$ yields

$$
\langle\sigma\rangle=\frac{I_{\mathrm{SD}}}{V_{\mathrm{SD}}} \frac{\ln \left(\frac{r_{\mathrm{a}}}{r_{\mathrm{i}}}\right)}{2 \pi}=\sigma_{\ell}+\frac{\alpha}{2 \pi}\left(\sigma_{h}-\sigma_{\ell}\right)<\sigma_{h}
$$

Thus, inhomogeneities acting as precursors of the filament would lead to subcritical $\sigma_{x x}$ values measured at values lower than predicted by the HEM. Instead, the experiments yield the opposite trend.

If we assume an additional 2D conduction mechanism to explain the higher saturation values of $\sigma_{x x}$, this would directly influence the measured hysteresis and is therefore less probable. It is more likely that the higher saturation values of the conductivity [although absolutely still small-a measured value of $\sigma_{x x}(\mathrm{sat})=6.7 \times 10^{-7} \mathrm{~S}$ corresponds to 8.6 $\left.\times 10^{-3} \sigma_{x y}\right]$ are due to parallel conduction in the GaAlAs doping layer of the heterostructure wafers. Such a parallel channel would only influence the measured value $\langle\sigma\rangle$, but neither $\sigma_{x x}$ in the 2DES nor the breakdown properties of the QHE in this system. This assumption is supported by the strong decrease of $\sigma_{x x}$ (sat) when decreasing the temperature below $1 \mathrm{~K}$ (see below).

To clarify the conduction mechanisms which contribute to $\sigma_{x x}$, we measured the temperature dependence in two different temperature ranges (between $2 \mathrm{~K}$ and $4 \mathrm{~K}$ in a $\mathrm{He}^{4}$ cryostat, and between $330 \mathrm{mK}$ and $1 \mathrm{~K}$ in a $\mathrm{He}^{3} / \mathrm{He}^{4}$ dilution refrigerator) at various fixed voltages $V_{\mathrm{SD}}$ below the critical breakdown value and fixed frequencies from 7 to $27 \mathrm{~Hz}$.

In Fig. 3, we see two different plots of $\sigma_{x x}(T)\left[\ln \sigma_{x x}\right.$ $=f\left(T^{-1}\right)$ assuming thermal activation, and $\ln \sigma_{x x}=f\left(T^{-1 / 2}\right)$ assuming $\mathrm{VRH}]$ for the two temperature ranges (wafer $A$ ). At temperatures above $2 \mathrm{~K}$, both plots yield reasonable linearity [Figs. 3(a) and 3(b)]. Consequently, TA and VRH are not 

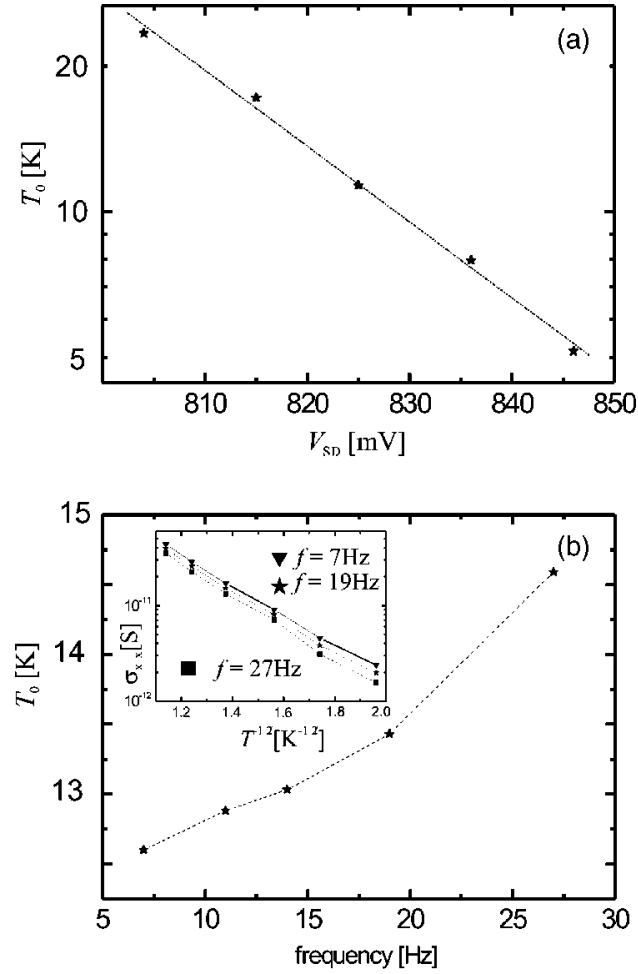

FIG. 4. Characteristic temperature $T_{0}$ obtained from VRH plot (see inset), (a) as a function of the subcritical dc voltage $V_{\mathrm{SD}}$ (70 $\mathrm{mK}<T<770 \mathrm{mK}$, sample $A 200)$ and (b) as a function of the frequency $(70 \mathrm{mK}<T<770 \mathrm{mK}$, sample $B 100$, subcritical ac amplitude $V_{\mathrm{SD}}=400 \mathrm{mV}$ ).

separable at these temperatures. However, at temperatures below $1 \mathrm{~K}$, the VRH plot yields a better linearity than the TA plot [Figs. 3(c) and 3(d)]. Thus, at these temperatures, VRH may be assumed to be dominant to determine $\sigma_{x x}$. The fact that the absolute values of $\sigma_{x x}$ are smaller than in Fig. 2 is due to the lower electric fields applied (the values given in Fig. 2 denote the amplitudes of the sinusoidal voltage) for the temperature-dependent measurements.

From the VRH plots, the values of the characteristic temperature $T_{0}$ can be deduced. Fig. 4 shows the dependence of $T_{0}$ on the applied voltage $V_{\mathrm{SD}}$ and on the frequency $f$. As expected, $T_{0}$ decreases markedly with increasing subcritical voltage [dc, see Fig. 4(a)]. This indicates a growing delocalization of the electrons with the voltage (already at subcritical voltages $V_{\mathrm{SD}}<V_{\mathrm{min}}$ ). The question may arise of whether a hopping conduction can be assumed to persist at rather high subcritical voltages of $V_{\mathrm{SD}} \sim 0.5-0.8 V_{\min }$, and whether a localization length $\xi$ can be deduced from the values of $T_{0}$ obtained at higher voltages. Rather few theoretical ${ }^{18,34}$ and experimental ${ }^{22,35,27}$ studies have addressed the problem of hopping conductivity in $\mathrm{QH}$ systems in the presence of higher electric fields. In these studies, the influence of the finite electric field $\boldsymbol{E}$ is described by an effective temperature $T_{\text {eff }}$, which is enhanced by the energy gain of the electrons in the electric field according to

$$
k_{\mathrm{B}} T_{\mathrm{eff}}=\frac{1}{2} e \boldsymbol{E} \xi
$$

Taking typical values of our experiment, $|\boldsymbol{E}|=4 \mathrm{kV} / \mathrm{m}$ and an estimated value of $|\boldsymbol{\xi}| \approx 10 \mu \mathrm{m}$ the decrease of $T_{0}$ as a function of $V_{\mathrm{SD}}$ [Fig. 4(a)] and the increase of $T_{0}$ as a function of the frequency [Fig. 4(b)] would correspond to an increase of $\xi$ from $4 \mu \mathrm{m}$ to $22 \mu \mathrm{m}$ and to a decrease of $\xi$ from $7.7 \mu \mathrm{m}$ to $6.6 \mu \mathrm{m}$, respectively, if Eq. (2b) were applicable\}, one obtains $T_{\text {eff }} \sim 200 \mathrm{~K}$. Such a temperature would not only terminate the hopping regime, but would also result in a complete breakdown of the QHE. However, as the QHE clearly persists at $|\boldsymbol{E}|>4 \mathrm{kV} / \mathrm{m}$ in our samples, the localization length $\xi$ as estimated from the VRH plots of $\sigma_{x x}$ vs temperature as in Fig. 3(d) cannot be correlated to the energy gain of the electrons. This is because the scattering properties of QH systems are highly anisotropic due to the Hall angle (between current density $\boldsymbol{j}$ and electric field $\boldsymbol{E}$ ) close to $90^{\circ}$. Under ideal QH conditions in a Corbino device, the radial voltage $V_{\mathrm{SD}}$ drives a circular current $I_{y}$, and $I_{\mathrm{SD}}=0$ holds. Assuming an average distance between two scattering events (most likely at Coulomb potentials of impurities ${ }^{26}$ ) along the drift direction of the electrons of the order of the localization length $\xi$, a single inelastic scattering event would displace the electrons, traveling on equipotential lines perpendicular to the radial field, radially by about the cyclotron diameter, $\Delta r \sim 2 R_{\mathrm{c}}$. In case of low Landau levels, $\Delta r$ is of the order of the magnetic length. Correspondingly, the theory of Fogler $e t$ $a l .{ }^{34}$ predicts a localization length approaching $2 R_{\mathrm{c}}$ in the center of a $\mathrm{QH}$ plateau. As it is difficult to measure the localization length at low electric fields at integer-filling factors, this prediction is hard to prove. However, the asymptotic behavior of $\xi(\nu)$ measured in Refs. 11 and 35 shows a trend of a reduction of $\xi$ towards $R_{\mathrm{c}}$ if $\nu$ approaches integer values. Thus, if we replace $\boldsymbol{\xi}$ in Eq. (4) by its component in the direction of the electric field, i.e., the magnetic length, we obtain an enhancement of the effective temperature of $\Delta T \sim 0.2-0.3 \mathrm{~K}$. This would explain the persistence of both the QHE and the VRH regime at bath temperatures below $1 \mathrm{~K}$.

The meaning of a quantity $\xi$ as obtained from a VRH plot according to Eq. (2b) at high electric fields remains a question if the scattering is highly anisotropic. As the order of this quantity corresponds to the typical drift distances between scattering events obtained in earlier studies, ${ }^{33} \xi(\mathrm{VRH})$ may stand for the unperturbed circular movement of electrons. Because this distance, at least for an assumed isotropic distribution of scatterers, has also to be overcome radially by the electrons to contribute to $I_{\mathrm{SD}}$, a corresponding value of $\xi$ may also determine the VRH plots of $\sigma_{x x}(T)$. However, this assumption would involve multiple scattering at impurities, displacing the electrons in radial direction by about $2 R_{\mathrm{c}}$ for each scattering event, without a complete loss of the phase of the wave function. To our knowledge, there is yet no complete theory of the anisotropic scattering properties in noneqilibrium $\mathrm{QH}$ systems available.

As mentioned above, the frequency dependence of the characteristic temperature $T_{0}$, obtained from $\sigma_{x x}(T)$ at different frequencies $7 \mathrm{~Hz}<f<27 \mathrm{~Hz}$, would correspond to a small decrease of the localization length $\xi$. The moderate decrease corresponds to the slight reduction of $\sigma_{x x}$ as shown in Fig. 2(d) for the same sample $B 100$ at $T<1 \mathrm{~K}$ in the same frequency range. (As mentioned above, measurements of $\sigma_{x x}$ 


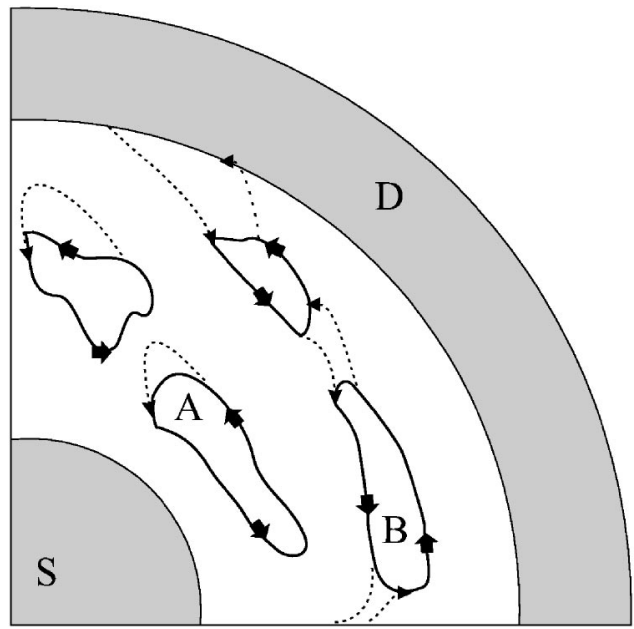

FIG. 5. Schematic picture of the suppression of the delocalization at low frequencies. Electrons start from the compressible islands and tunnel towards neighboring compressible islands. The electric field changes sign before the compressible islands start to overlap (ac, trajectory $A$, no contribution to source-drain current). If the electric field is permanently applied, the electrons accomplish their way between the electrodes via the compressible islands ( $\mathrm{dc}$, trajectory $B$, finite source-drain current).

below $7 \mathrm{~Hz}$ were technically not possible for $T<1 \mathrm{~K}$.) From the results we conclude that the delocalization in the $\mathrm{QH}$ system becomes less effective in comparison to the dc case if alternating electric fields with high but subcritical amplitudes are applied.

In Fig. 5, we show a scheme of a possible scenario to explain the effect: the closed loops represent localized states (compressible islands) inside the $\mathrm{QH}$ system. If a constant electric field is applied or the frequency is sufficiently low, electrons tunnel at a rather high rate between the compressible islands, which increases by this process. ${ }^{36,37}$ Thus, with a certain probability they can be transferred from the source $(S)$ to the drain $(D)$ contact via these compressible islands (trajectories $B$ in Fig. 5). If an alternating electric field is applied, the electrons start from any compressible islands and tunnel at a certain rate towards the neighboring compressible islands electrode via the localized states during the presence of one half wave $t_{1 / 2}$ of the electric field. If this half wave $t_{1 / 2}$ is short enough, the polarity of the ac field changes before the compressible states start to overlap (trajectories of type $A$ in Fig. 5). The trajectories of type $A$ contribute nothing to the source-drain current and thus to $\sigma_{x x}{ }^{36,37}$ Furthermore, a redistribution of the compressible islands themselves can be assumed to be responsible for the delocalization of electrons at high electric fields.

As the steep drop of $\sigma_{x x}$ occurs typically at about $10 \mathrm{~Hz}$ (please note that the drop in Fig. 2(d) is by orders of magnitude smaller than in Figs. 2(a)-2(c) and represents the very tiny changes within the saturation of $\sigma_{x x}$ ), the time $t_{1 / 2}$ $=1 /(2 f)$ is of the order of $50 \mathrm{~ms}$. This appears rather long in view of the delocalization times of a few nanoseconds as measured in pulsed breakdown experiments. ${ }^{33}$ However, the measurements presented here are performed at subcritical electric fields of $E_{\mathrm{SD}} / E_{\mathrm{c}} \approx 0.5\left(E_{\mathrm{c}}\right.$ is the critical electric field for the breakdown of the QHE), whereas the data in Ref. 33 were taken at $1.2<E_{\mathrm{SD}} / E_{\mathrm{c}}<3$. The delocalization times are determined basically by the transition times between compressible islands or the redistribution of those, which are a strong function of the applied field and of the temperature. (Time scales for the redistribution of charges between localized states up to about $20 \mathrm{~min}$ at $\mathrm{mK}$ temperatures have been observed ${ }^{38}$ ) The dissipative current density $j_{x}$ related to trajectories of the type $B$ is quite small. A simple estimate of $j_{x}=n_{\mathrm{s}} e \xi\left(T_{\mathrm{el}}, E_{\mathrm{SD}}, f\right) / t_{1 / 2}$ yields $1.2 \times 10^{-7} \mathrm{~A} / \mathrm{m}$ for the saturation current density for sample $B 100$ at $T_{\mathrm{el}}<1 \mathrm{~K}, E_{\mathrm{SD}} / E_{\mathrm{c}}$ $\approx 0.5, f>10 \mathrm{~Hz}$, far smaller than the corresponding circular current density $j_{y}=\sigma_{x y} E_{\mathrm{SD}} \approx 0.3 \mathrm{~A} / \mathrm{m}$. The saturation value of $\sigma_{x x}=2.9 \times 10^{-11} \mathrm{~S}$ from the estimate above agrees suprisingly well with the saturation value measured for this sample at $T=0.77 \mathrm{~K}, f=27 \mathrm{~Hz}$, and $V_{\mathrm{SD}}=0.4 \mathrm{~V}$. In view of the enhanced saturation values as discussed above, this could indicate a strong reduction of the parallel conduction in the doping layer when reducing the temperature from $1.6 \mathrm{~K}$ to $0.77 \mathrm{~K}$.

\section{SUMMARY}

In previous measurements, we have observed a steep increase of the dynamical hysteresis of the breakdown of the QHE with increasing frequency up to $10-20 \mathrm{~Hz}$. This increase stems from an increase of the upper hysteresis limit $V_{\max }$ at an almost unchanged lower hysteresis limit $V_{\min }$ (see Ref. 25). We could predict such a behavior within a hotelectron model (HEM) by the assumption of a background conductivity $\sigma_{\mathrm{BG}}$ of the $\mathrm{QH}$ system which drops already at low frequencies. In this study, we proved this by measurements of the conductivity $\sigma_{x x}$ (at voltages far from equilibrium, but below the critical voltage for the breakdown of the QHE) of GaAs/GaAlAs Corbino devices in the quantum Hall $(\mathrm{QH})$ regime at low frequencies from dc up to $10 \mathrm{kHz}$. At very low frequencies up to $10-20 \mathrm{~Hz}, \sigma_{x x}$ shows a steep decrease from its dc value towards a reduced saturation value. This decrease of $\sigma_{x x}$ is of the same order as predicted by the HEM. Measurements of the temperature dependence of the nonequilibrium $\sigma_{x x}$ from $70 \mathrm{mK}$ to $4.2 \mathrm{~K}$ revealed a dominance of a background conductivity with a temperature-dependence-like variable-range hopping (VRH) $\sigma_{x x} \sim \exp \left\{-\left(T_{0} / T\right)^{1 / 2}\right\}$ for temperatures below $1 \mathrm{~K}$ and an additional contribution of thermal activation above $2 \mathrm{~K}$. This is a clear evidence that the conductivity at subcritical voltages, in particular the background contribution $\sigma_{\mathrm{BG}}$, directly affects the maximum breakdown limit $V_{\max }$ of the QHE. This background contribution, and thus $V_{\max }$, are therefore dominated by low-energy excitations between compressible islands. In contrast, the lower limit $V_{\min }$ of the hysteresis is determined by activation processes from localized to extended states. The characteristic temperature $T_{0}$ as determined from VRH-like temperature plots of the conductivity also showed an increase with the frequency. We attribute these results to a less-effective delocalization of electrons in alternating external fields with increasing frequency, due to a reduced heating and scattering between compressible islands. 
The time scale for the additional ac localization is rather long $(50-100 \mathrm{~ms})$ and determines the saturation limit of the nonequilibrium conductivity $\sigma_{x x}$ of the $\mathrm{QH}$ system.

From the results we can conclude for our samples that the breakdown stability of the ac QHE at low frequencies is distinctively higher than in the DC case.

\section{ACKNOWLEDGMENTS}

This work was supported by the Deutsche Forschungsgemeinschaft (DFG, Grant No. Na235/10-2,3). The wafers investigated in this study were provided by the MBE group of the Max-Planck Institut für Festkörperforschung, Stuttgart (Germany). *g.nachtwei@tu-bs.de

${ }^{1}$ E. Abrahams, P. W. Anderson, D. C. Licciardello, and T. V. Ramakrishnan, Phys. Rev. Lett. 42, 673 (1979).

${ }^{2}$ H. Aoki and T. Ando, Phys. Rev. Lett. 54, 831 (1985).

${ }^{3}$ A. M. M. Pruisken, Phys. Rev. Lett. 61, 1297 (1988).

${ }^{4}$ B. Huckestein, Rev. Mod. Phys. 67, 357 (1995).

${ }^{5}$ S. L. Sondhi, S. M. Girvin, J. P. Carini, and D. Shahar, Rev. Mod. Phys. 69, 315 (1997).

${ }^{6}$ H. P. Wei, D. C. Tsui, M. A. Paalanen, and A. M. M. Pruisken, Phys. Rev. Lett. 61, 1294 (1988).

${ }^{7}$ L. W. Engel, H. P. Wei, D. C. Tsui, and M. Shayegan, Surf. Sci. 229, 13 (1990).

${ }^{8}$ S. Koch, R. J. Haug, K. von Klitzing, and K. Ploog, Phys. Rev. B 43, R6828 (1991); Phys. Rev. Lett. 67, 883 (1991).

${ }^{9}$ L. W. Engel, D. Shahar, Ç. Kurdak, and D. C. Tsui, Phys. Rev. Lett. 71, 2638 (1993).

${ }^{10}$ F. Hohls, U. Zeitler, R. J. Haug, R. Meisels, K. Dybko, and F. Kuchar, Phys. Rev. Lett. 89, 276801 (2002).

${ }^{11}$ F. Hohls, U. Zeitler, and R. J. Haug, Phys. Rev. Lett. 86, 5124 (2001).

${ }^{12}$ G. Ebert, K. von Klitzing, K. Ploog, and G. Weimann, J. Phys. C 16, 5441 (1983).

${ }^{13}$ S. Komiyama, T. Takamasu, S. Hiyamizu, and S. Sasa, Solid State Commun. 54, 479 (1985).

${ }^{14}$ G. Nachtwei, Z. H. Liu, G. Lütjering, R. R. Gerhardts, D. Weiss, K. von Klitzing, and K. Eberl, Phys. Rev. B 57, 9937 (1998).

${ }^{15}$ S. Komiyama and Y. Kawaguchi, Phys. Rev. B 61, 2014 (2000).

${ }^{16}$ Y. Ono, J. Phys. Soc. Jpn. 51, 237 (1982).

${ }^{17}$ G. Ebert, K. von Klitzing, C. Probst, E. Schuberth, K. Ploog, and G. Weimann, Solid State Commun. 45, 625 (1983).

${ }^{18}$ D. G. Polyakov and B. I. Shklovskii, Phys. Rev. Lett. 70, 3796 (1993); Phys. Rev. B 48, 11167 (1993).

${ }^{19}$ S. Kawaji and K. Wakabayashi, Solid State Commun. 22, 87
(1977).

${ }^{20}$ R. J. Nicholas, R. A. Stradling, and R. J. Tidey, Solid State Commun. 23, 341 (1977).

${ }^{21}$ Th. Englert and K. von Klitzing, Surf. Sci. 73, 70 (1978).

${ }^{22}$ P. Svoboda, G. Nachtwei, C. Breitlow, S. Heide, and M. Cukr, Semicond. Sci. Technol. 12, 264 (1997).

${ }^{23}$ J. Schurr, J. Melcher, A. von Camphausen, G. Hein, F.-J. Ahlers, and K. Pierz, Metrologia 39, 3 (2002).

${ }^{24}$ G. Nachtwei, N. K. Kalugin, B. E. Sağol, A. Buß, and Ch. Stellmach, Appl. Phys. Lett. 82, 2068 (2003).

${ }^{25}$ N. G. Kalugin, B. E. Sağol, A. Buss, A. Hirsch, Ch. Stellmach, G. Hein, and G. Nachtwei, Phys. Rev. B 68, 125313 (2003).

${ }^{26}$ I. I. Kaya, G. Nachtwei, K. von Klitzing, and K. Eberl, Phys. Rev. B 58, R7536 (1998).

${ }^{27}$ F. Hohls, U. Zeitler, and R. J. Haug, Phys. Rev. Lett. 88, 036802 (2002).

${ }^{28}$ N. F. Mott, J. Non-Cryst. Solids 1, 1 (1968).

${ }^{29}$ A. L. Efros and B. I. Shklovskii, J. Phys. C 8, L49 (1975).

${ }^{30}$ G. Nachtwei, Physica E (Amsterdam) 4, 79 (1999).

${ }^{31}$ W. Eberle, J. Hirschinger, U. Margull, W. Prettl, V. Novak, and H. Kostial, Appl. Phys. Lett. 68, 3329 (1996).

${ }^{32}$ K. Aoki and S. Fukui, Physica B 272, 274 (1999).

${ }^{33}$ B. E. Sağol, G. Nachtwei, K. von Klitzing, G. Hein, and K. Eberl, Phys. Rev. B 66, 075305 (2002).

${ }^{34}$ M. M. Fogler, A. Yu. Dobin, and B. I. Shklovskii, Phys. Rev. B 57, 4614 (1998).

${ }^{35}$ M. Furlan, Phys. Rev. B 57, 14818 (1998).

${ }^{36}$ V. Tsemekhman, K. Tsemekhman, C. Wexler, J. H. Han, and D. J. Thouless, Phys. Rev. B 55, R10 201 (1997).

${ }^{37}$ K. Güven, R. R. Gerhardts, I. I. Kaya, B. E. Sağol, and G. Nachtwei, Phys. Rev. B 65, 155316 (2002).

${ }^{38}$ Yayi Wei, Ph.D. thesis, University of Stuttgart, 1998, p. 78. 